\title{
CMapped: Um Ambiente Flexível Baseado em Mapas Conceituais
}

\author{
Walber Antonio Ramos Beltrame ${ }^{1}$ \\ ${ }^{1}$ Instituto Federal do Espírito Santo (IFES) \\ Caixa Postal S/N - 29.215-090 - Guarapari - ES - Brasil \\ $\{$ walber.beltrame egmail.com
}

\begin{abstract}
Resumo. Este trabalho apresenta um ambiente voltado à aprendizagem por meio da organização de conteúdos didáticos, da construção de saberes e de conhecimento, do estímulo às atividades cooperativas e do exercício da reconstrução cognitiva do aprendiz, utilizando-se de uma interface flexível baseada em Mapas Conceituais. O objetivo da ferramenta é o suporte a modelos pedagógicos abertos e a realização de tarefas planejadas, com o mérito de considerar que, tanto os participantes quanto os conteúdos, podem ser representados como conceitos de um mapa. A navegação e a interação entre os conceitos e relações do mapa é a característica principal do ambiente, como um facilitador dos processos educacionais.
\end{abstract}

\section{Cenário de Uso}

O uso de Ambientes Virtuais de Aprendizagem no cenário da Internet é o motivador de diversos trabalhos e pesquisas sobre como elaborar ferramentas que ofereçam suporte a educação a distância e apoio a atividades presenciais de ensino e de aprendizagem. $\mathrm{O}$ objetivo deste trabalho é apresentar a versão inicial de um sistema $W e b$, decorrente da proposta de um ambiente baseado em Mapas Conceituais [Novak 1990], denominado de CMapped [Beltrame 2014].

Este ambiente de aprendizagem visa a apropriação da tecnologia para o suporte às teorias da aprendizagem cognitivistas, segundo correntes de psicologia significativa [Ausubel 1963], interacionista [Vygotsky 1978], construtivista [Piaget 1977] e autômata [Freire 1998]. Em específico, espera-se estimular abordagens pedagógicas da incerteza [Carvalho et al. 2005], em que o aluno é incentivado a lidar com convicções provisórias e com dúvidas que o faça perceber novas convicções. Desse modo, um dos objetivos é oferecer apoio telemático a Arquiteturas Pedagógicas [Carvalho et al. 2005], que é uma proposta recente para se trabalhar com ambientes virtuais (ver Seção 3).

A fim de atingir esses propósitos, o princípio do CMapped, enquanto Ambiente Virtual de Aprendizagem, é que todos conteúdos e interações possam estar relacionados, como um grande repositório de Mapas Conceituais. Os conceitos são representações de conteúdo, em formatos multimídias, como textos, imagens, vídeos, páginas, arquivos e objetos de aprendizagem. O ponto principal do CMapped é tratar os participantes também como conceitos. A ideia do ambiente é fazer a gestão do compartilhamento dos mapas por meio de adicionar ou não o participante, na forma de conceito. Ao iniciá-lo com a letra “@”, acrescida do nome de quem se quer compartilhar, passa-se a ter direito a visualização, mas somente dos conceitos relacionados, semelhante à definição de caminho em teoria dos grafos. Se existir uma relação também iniciada com a letra "@”, permite-se alterações nos conceitos e criação de novos. 
Ao iniciar um conceito do tipo texto com a letra "+”, significa para o CMapped a criação de mapa, sendo esse o atalho para a página do novo Mapa Conceitual. Nesse novo mapa, o participante poderá fazer novos conceitos, relações e mapas (Figura 1). Desse modo, pode-se criar e navegar por diversos mapas. Todo participante possui também o mapa relacionado ao nome, que é acessado ao digitá-lo no final do endereço $W e b$ do sistema, por meio do navegador de Internet. É possível, para cada mapa, definir um nome para o endereço, na forma de link, para facilitar o acesso (ver Seção 3).

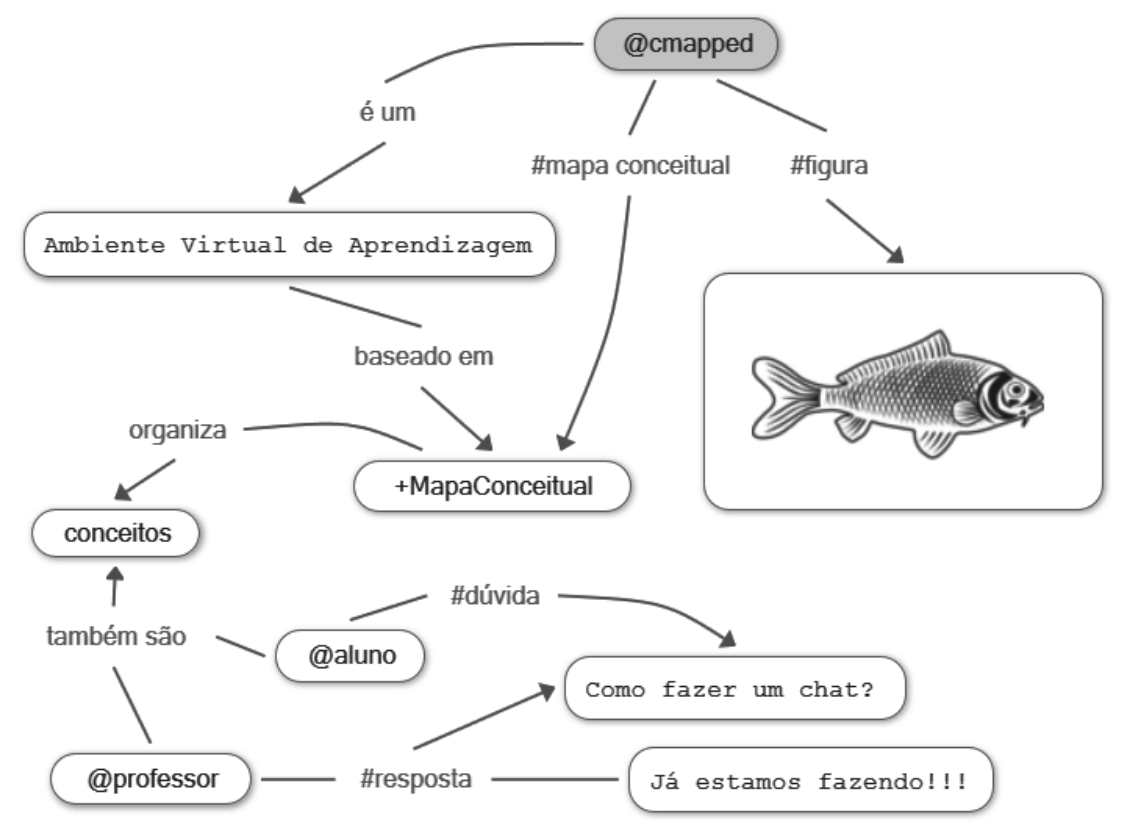

Figura 1. Exemplo de Mapa Conceitual no CMapped [Beltrame 2014]

Essas simples estratégias permitem que os conteúdos produzidos na forma de mapas possam ser facilmente compartilhados. Não obstante à facilidade, passa-se o aluno também a ser um dos protagonistas do aprendizado, à medida que o permite construir e evoluir o mapeamento das informações, estimulado pelos professores, por exemplo, as anotações feitas em sala, as soluções de problemas e a representação do conhecimento sobre determinado assunto, aliado ao esforço cognitivo de relacionar os conceitos. No modo compartilhado, estimula-se grupos de estudantes a ter entendimento comum da matéria, com didáticas apropriadas.

Cabe ao professor planejar corretamente as atividades, podendo utilizar-se do próprio ambiente para essa tarefa. Novak e Canãs (2006) propõe o uso de ferramentas ao encontro da aprendizagem significativa, contrário a atividades mecanizadas de ensino, para qualquer nível de aprendizagem. Para acomodar os diferentes perfis de estudantes, do ensino fundamental ao superior, buscou-se também no CMapped uma interface intuitiva e minimalista, de arrastar e soltar, de modo que os alunos se concentrem nas tarefas de relacionar e de demonstrar. Outras funções úteis também foram implantadas, como filtro e histórico de alterações (ver Seção 3). Destaca-se o aspecto generalista e multidisciplinar da proposta.

Nesse contexto, ressalta-se a modelagem de Arquiteturas Pedagógicas, que são propostas de ensino embasadas, com metas, planejamento e articulação. Por iniciativa desse texto, propõe-se o CMapped como um ferramental capaz de desenvolvê-las. Para Carvalho et al. (2005), as arquiteturas são como mapas ao mostrar diferentes direções para se realizar algo, condizente com o que se espera do sistema (ver Seção 3.1). 
CBIE-LACLO 2015

Anais dos Workshops do IV Congresso Brasileiro de Informática na Educação (CBIE 2015)

\section{Desenvolvimento}

O processo de desenvolvimento do sistema seguiu o modelo de software livre e código aberto $^{1}$, utilizando-se de padrões e de tecnologias que seguem esse método. Espera-se a formação de uma comunidade de colaboradores, a partir da divulgação e utilização do ambiente. O padrão arquitetural da tecnologia foi projetado para suportar a estrutura da $W e b$, seguindo a definição do tipo cliente-servidor em três camadas, segundo o padrão modelo-controle-visão.

$\mathrm{Na}$ camada de interface no cliente (navegador $W e b$ ), foi utilizada a biblioteca jsPlumb ${ }^{2}$ para criação das relações entre os conceitos. Para os demais controles, como botões e janelas, foi utilizado a biblioteca JQuery ${ }^{3}$. Para alguns tipos de conceitos, outras extensões do JQuery foram utilizadas, todas de projetos de permissão livre.

De tempos em tempos e para cada alteração no Mapa Conceitual, requisições do tipo Ajax ${ }^{4}$ são enviadas para servlets da tecnologia Java Server Faces ${ }^{5}$ no servidor de aplicação, que faz a sincronização e persistência das alterações por meio de um framework orientado a grafo Titan ${ }^{6}$. O Titan é uma biblioteca transparente ao sistema de banco de dados, suportando as plataformas livres: Oracle BerkeleyDB ${ }^{7}, \mathrm{Apache} \mathrm{Hbase}^{8}$, Apache Cassandra ${ }^{9}$.

Para comunicação entre o servidor de aplicação e a camada do cliente, os dados são anotados utilizando-se a biblioteca $X_{S} \operatorname{Stream}^{10}$ na linguagem $X M L$ e indexados na plataforma Apache Lucene ${ }^{11}$, evitando conexões em excesso ao banco de dados. Foi definido também uma estrutura de cache, utilizando-se a tecnologia Apache JCS ${ }^{12}$.

Guarda-se o endereço de imagens, vídeos e páginas na Internet, sendo um texto do link para esses elementos $W e b$, o que não demanda muito espaço em disco. No entanto, permite-se criar conceitos do tipo arquivo. Nesses casos, utiliza-se para guarda dos documentos a biblioteca de arquivos Apache Hadoop ${ }^{13}$. A ferramenta permite a utilização de sistemas distribuídos, mas é necessário configuração.

Na próxima seção é descrito a validação e avaliação técnica do sistema e das bibliotecas, por meio de estudo com três grupos de aproximadamente quarenta alunos, descrevendo-se os recursos de hardware mínimos testados.

$1 \mathrm{http}: / /$ github.com/cmapped

2 http://www.jsplumb.org

3 http://jquery.com

4 http://ajaxpatterns.org

$5 \mathrm{http} / / /$ javaserverfaces.java.net/

6 http://thinkaurelius.github.io/titan/

7 http://www.oracle.com/technetwork/database/database-technologies/berkeleydb

8 http://hbase.apache.org/

9 http://cassandra.apache.org

$10 \mathrm{http}: / / \mathrm{x}$-stream.github.io/

11 http://lucene.apache.org/

$12 \mathrm{http} / /$ commons.apache.org/proper/commons-jcs/

13 http://hadoop.apache.org/ 
CBIE-LACLO 2015

Anais dos Workshops do IV Congresso Brasileiro de Informática na Educação (CBIE 2015)

\subsection{Validação}

Para realizar-se a primeira validação inicial do ambiente, estruturou-se um servidor com os requisitos mínimos de hardware para um sistema $W e b$ da plataforma Java, sendo esse estabelecido com: sistemas operacional Ubuntu Server 14 LTS 64-bit, dois núcleos de processamento Intel Xeon virtualizados na tecnologia VMWare, máquina virtual Java 7 e servidor de aplicação Apache Tomcat 7. Configurou-se o sistema para se utilizar o banco de dados Oracle BerkeleyDB e sistemas de arquivos local, todos de forma não distribuída (única máquina para atender a todas requisições).

O estudo de caso escolhido para testes foi a aplicação da Arquitetura Pedagógica Debate de Teses [Nevado et al. 2011] (ver Seção 3.1), para a disciplina Informática Básica, no mês inicial do ano letivo, totalizando 124 alunos (em média 40 alunos por sala), para três turmas do primeiro ano do Ensino Médio integrado aos cursos técnicos de Administração, Mecânica e Eletrotécnica do Instituto Federal do Espírito Santo, campus Guarapari, mesclando atividades em sala de aula e para casa. A idade dos alunos variava entre 14 e 16 anos, sendo que, após rápida pesquisa, fora o primeiro contato dos estudantes com um Ambiente Virtual de Aprendizagem.

Na primeira atividade, após explicação inicial do ambiente em sala, os alunos foram incentivados a escreverem o perfil pessoal. Nesse momento, o sistema apresentou instabilidade, devido a falhas de configuração do servidor Tomcat, o que fora corrigido para as próximas semanas. Durante as outras atividades, recomendou-se aos estudantes observarem falhas e sugestões de melhoria. Realizou-se acompanhamento dos $\log s$ do sistema. Para cada anotação, categorizou-se em defeito ou sugestão, se a urgência era grave à baixa, discutindo-se possíveis tarefas a realizar para as próximas versões. Esse procedimento está sintetizado na Tabela 1.

Tabela 1. Validação inicial e tarefas a realizar

\begin{tabular}{|c|c|c|c|c|}
\hline & Relatos e sugestões & Tipo & Urgência & Tarefas a realizar \\
\hline 1 & $\begin{array}{l}\text { O sistema apresentava } \\
\text { mensagem de erro ao se } \\
\text { acessar pelo endereço iniciado } \\
\text { com } w w w \text {. }\end{array}$ & defeito & médio & $\begin{array}{l}\text { As requisições do tipo Ajax } \\
\text { possuem regra de segurança } \\
\text { nos navegadores se o } \\
\text { endereço da chamada for } \\
\text { diferente do estabelecido no } \\
\text { Javascript. É necessário } \\
\text { configurar o servidor para } \\
\text { redirecionar o endereço para } \\
\text { o que é configurado no } \\
\text { sistema. Feito isso, a falha é } \\
\text { corrigida. }\end{array}$ \\
\hline 2 & $\begin{array}{l}\text { Após uso intenso em sala de } \\
\text { aula, o sistema parava de } \\
\text { funcionar. Verificou-se no log } \\
\text { do Tomcat o erro: } \\
\text { java.lang. OutOfMemoryError } \\
\text { PermGen space. }\end{array}$ & defeito & grave & $\begin{array}{l}\text { É necessário configurar o } \\
\text { servidor Tomcat para } \\
\text { suportar um número de } \\
\text { memória maior do que o } \\
\text { padrão para a máquina } \\
\text { virtual Java. Disponibilizou- } \\
\text { se } 1024 \text { MB e a falha não } \\
\text { voltou a ocorrer. }\end{array}$ \\
\hline
\end{tabular}


Foi configurado somente a permissão do tamanho dos arquivos para $5 \mathrm{MB}$. Para atividades educacionais, esse

3 número pode ser insuficiente para a demanda. Há também um aumento no número de memória no momento da submissão de arquivos, devido que o sistema os compacta.

Alguns alunos relataram que os conectores das relações

4 ficavam invisíveis, aparecendo somente as setas. Após se configurar a cor, volta-se ao normal.

Muitos alunos solicitaram que queriam apagar os conceitos e relações e começar tudo do zero, mas o sistema não tem essa opção, somente deixar os elementos invisíveis.

Ao carregar o sistema na

6 primeira vez, há uma certa rápido. demora. Nas outras vezes ele é defeito baixo sugestão baixo

defeito médio

sugestão baixo
Dependendo do número de alunos e dada a liberdade de se enviar quantos arquivos se deseja, é necessário avaliar a capacidade do sistema de arquivos, antevendo um grande número de uploads. Aquivos maiores agravariam o problema. Se realmente necessários, recomenda-se usar um serviço externo de guarda de arquivos.

É necessário investigar em quais navegadores essa situação ocorreu. Manter a biblioteca jsPlumb atualizada é recomendado. A falha está sob análise.

Tal característica foi definida de forma proposital, até pelo fato de o professor querer analisar todo o histórico de alteração do mapa. Deixar invisível é uma forma de exclusão.

Por utilizar de uma interface rica, o tamanho das bibliotecas Javascript gira em torno de 2 MB. Depois de carregadas elas funcionam em cache. Portanto, o comportamento é normal.

Será realizado um estudo de interface do ambiente para avaliar a possibilidade de se

Na função de histórico, poderia a exibição ser mais parecida com um filme. Não é exibido

7 uma listagem do que fora realizado, é possível somente acompanhar as alterações de forma visual.

O posicionar e reposicionar do

8 histórico às vezes não exibe o defeito médio mapa de forma correta

Há um aumento considerável

9 do tráfego de rede no momento defeito baixo da atividade em sala. exibir a lista de alterações do mapa e a exibição do histórico de forma mais suave (trabalhos futuros).

$\mathrm{O}$ algoritmo de histórico deverá ser revisto para a próxima versão.

Por analisar as alterações a cada instante, esse comportamento é normal. 
CBIE-LACLO 2015

Anais dos Workshops do IV Congresso Brasileiro de Informática na Educação (CBIE 2015)

\section{Apresentação do software}

O ambiente foi continuado e aprimorado da proposta de Beltrame (2014). É definido uma interface minimalista, em que há uma tela de trabalho com três funções principais: arrastar conceitos (quinze tipos de formatos multimídias) para o Mapa Conceitual, um filtro de visualização de conceitos no mapa e uma interface de visualização de histórico de alterações (Figura 2).

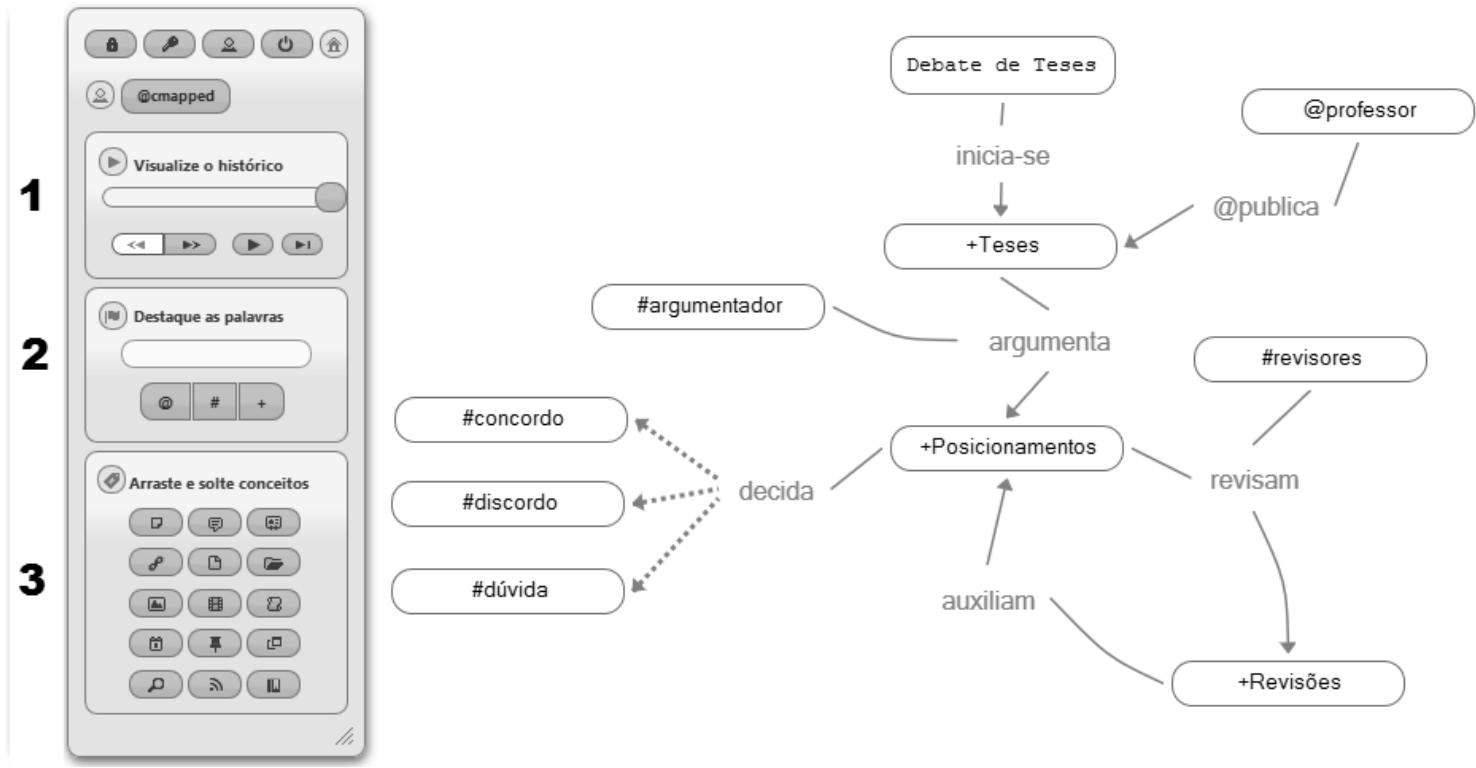

Figura 2. Tela do CMapped e descrição do Debate de Teses

No restante da tela, visualiza-se e se edita o Mapa Conceitual, da seguinte forma: arrasta-se os conceitos para uma posição do mapa, redimensiona-se (ícone a direita inferior), cria-se relações entre os conceitos (ícone a esquerda inferior), sendo que alguns conceitos é necessário edição em tela separada (ícone a direita superior). É possível editar a borda (cor, espessura, formato), a fonte dos textos e o plano de fundo. O mapa e os conectores também são editáveis, para tanto, clica-se com o botão direito do mouse nos elementos e um novo menu, com as opções apropriadas, é exibido.

Dos quinze conceitos possíveis (Destaque 3 da Figura 2), o primeiro é referente a um termo ou palavra, ou seja, não é possível escrever um texto (com mais de duas palavras). Com ele, trabalha-se a questão das permissões no mapa, em relação aos outros usuários, usando-se o caractere "@”. Outro caractere importante é o "\#”, que será como dizer que o conceito terá um destaque no Mapa. Assim, pode-se filtrar a exibição dos termos que possuem o caractere ou não (Destaque 2 da Figura 1), o mesmo vale para o “@”, e o “+”. Esse último indica ao sistema que o conceito é um atalho para outro novo Mapa Conceitual, dessa forma pode-se navegar entre diferentes mapas, como um link. É permitido voltar e gerenciar links, usando-se as opções do menu do mapa.

Além do conceito palavra, foram desenvolvidos na versão inicial quatorze outros tipos: texto, hipertexto, link, arquivo, pastas, arquivos, figura, vídeo, código, calendário, mapa geográfico, janela, pesquisa, disseminação e relatórios. Outro recurso importante para o processo de avaliação é o histórico de versões (Destaque 3 da Figura 1). Aliado as ferramentas de filtro, pode-se saber exatamente qual foi o processo de construção e reconstrução do mapa, sendo importante mecanismo de acompanhamento do processo cognitivo dos estudantes. 
CBIE-LACLO 2015

Anais dos Workshops do IV Congresso Brasileiro de Informática na Educação (CBIE 2015)

\subsection{Avaliação}

A metodologia usada para avaliação inicial da ferramenta seguiu-se o planejamento de atividades da Arquitetura Debate de Teses, adaptada para o cenário do CMapped: (1) o professor explicou a arquitetura e o que os papéis dos alunos; (2) foi exposto teses simples sobre Informática Básica, por exemplo, "computadores menores, como tablets, não possuem placa-mãe"; (3) os alunos tinham que criar um mapa conceitual para cada tese, em sala de aula, para se posicionar a favor, contra ou com dúvida, inserindo figuras, vídeos e links para fortalecer a argumentação; (4) como atividade para casa, o professor compartilhou com outro dois alunos o mapa, que teriam o papel de fazer revisão; (5) $\mathrm{O}$ aluno deveria observar as mudanças dos colegas e alterar o mapa de acordo com as sugestões; (6) Por fim, foi realizada discussão e apresentação de alguns mapas para toda a turma.

Um dos objetivos principais da avaliação fora verificar se o ambiente atende aos requisitos básicos para articulação (1) e coordenação (2) de Arquiteturas Pedagógicas [Vieira Jr. et al. 2011], resumidos na Tabela 2. É possível realizar as atividades básicas, no entanto, algumas ações dependem mais do esforço e explicação do professor do que uma regra fixa no sistema, por exemplo, cumprir prazos e definir papéis para leitura e escrita. Tal característica minimiza as necessidades de configuração, dado que o sistema preza pela facilidade, mas o torna permissivo, ou seja, difícil para definição de regras. Outro objetivo do trabalho é a análise qualitativa da ferramenta, o esforço de usabilidade e de plasticidade por parte dos alunos quanto a interface simplista e a descoberta das possibilidades e das dificuldades do sistema. Porventura, os detalhes desses propósitos nortearão futuras publicações sobre o ambiente, ao ponto que se aguarda a formação de uma comunidade entusiasta e colaboradora.

Tabela 2. Avaliação de Requisitos do CMapped quanto a Arquiteturas Pedagógicas

\begin{tabular}{|l|l|l|}
\hline \multicolumn{2}{|c|}{ Requisitos } & \multicolumn{2}{c|}{ Observação } \\
\hline C.E.R & 3 & Cada mapa é um espaço reservado \\
\hline Convidar usuários & 3 & Para cada mapa, adicionam-se usuários \\
\hline Determinação de Papéis & 2 & Depende da explicação do professor \\
\hline Dependência de Atividades & 2 & Depende da explicação do professor \\
\hline Restrição de Interações & 3 & Permissão de leitura e escrita para cada mapa \\
\hline Perfis Dinâmicos & 2 & Depende da explicação do professor \\
\hline Definir Permissões de Leitura & 3 & Permissão de leitura e escrita para cada mapa \\
\hline Definir Prazo de Leitura & 2 & Depende da atuação do professor \\
\hline Definir Permissões de Escrita & 3 & Permissão de leitura e escrita para cada mapa \\
\hline Definir Prazo de Escrita & 2 & Depende da atuação do professor \\
\hline Multiplicidade de Papeis & 2 & Depende da explicação do professor \\
\hline Redefinir Permissões de Leitura & 3 & Permissão de leitura e escrita para cada mapa \\
\hline Redefinir Prazo de Leitura & 2 & Depende da atuação do professor \\
\hline Redefinir Permissões de Escrita & 3 & Permissão de leitura e escrita para cada mapa \\
\hline Redefinir Prazo de Escrita & 2 & Depende da atuação do professor \\
\hline
\end{tabular}


CBIE-LACLO 2015

Anais dos Workshops do IV Congresso Brasileiro de Informática na Educação (CBIE 2015)

\section{Considerações finais}

$\mathrm{O}$ aspecto inovador do ambiente CMapped encontra-se na premissa que os processos educacionais podem ser realizados pela disposição de conteúdos relacionados, como Mapas Conceituais. Desse modo, um facilitador para a tarefa de delegar permissão de leitura e de escrita é considerar os participantes como conceitos do mapa.

A validação da ferramenta encontra-se em estágio introdutivo. Um dos méritos deste artigo é a divulgação da análise inicial realizada e a verificação que a ferramenta atende aos requisitos formais de se trabalhar com propostas inovadoras e abertas, com um estudo de caso referente ao suporte telemático de Arquiteturas Pedagógicas.

Há diferentes oportunidades quanto a pesquisas e contribuições para o sistema e a política de código aberto e livre reflete essa expectativa. Pode-se investigar outros campos de utilização da ferramenta, como gestão de conhecimento. Os trabalhos futuros poderão também observar o aspecto computacional provido. Dentre as possibilidades, listam-se: comparação de mapas, avaliação automática, geração automática de mapas a partir de páginas da internet, criação de serviços de consulta e resposta automática.

\section{Referencias}

Ausubel, D. P. (1963). The Psychology of Meaningful Verbal Learning. New York: Grune and Stratton.

Beltrame, W.A.R. (2014). CMapped: Uma Proposta de Ambiente Virtual de Aprendizagem Baseado em Mapas Conceituais. Anais da VI Conference on Concept Mapping.

Carvalho, M. J. S.; Nevado, R. A.; Menezes, C. S. (2005). Arquiteturas Pedagógicas para Educação a Distância: Concepções e Suporte Telemático. Anais do XVI Simpósio Brasileiro de Informática na Educação.

Freire, P. (1998) Pedagogia da Autonomia: saberes necessários à prática educativa. Rio de Janeiro: Paz e Terra.

Nevado, R. A.; Menezes, C. S.; Vieira Júnior, R. R. M. (2011). Debate de Teses: uma arquitetura pedagógica. Anais do XXII Simpósio Brasileiro de Informática na Educação.

Novak, J. D. (1990). Concept maps and Vee diagrams: Two metacognitive tools to facilitate meaningful learning. Instructional science, 19(1), 29-52.

Novak, J. D.; Cañas, A. J. (2006). The Theory Underlying Concept Maps and How to Construct Them. Technical report. Florida: Institute for Human and Machine Cognition.

Piaget, J. (1977). Psychology and epistemology: Towards a theory of knowledge. New York: Penguin Books.

Vieira Jr., R. R. M.; Santos, O. L.; Rafalski, J. P.; Bada, E. M.; Silva, H. F. A.; Menezes, C. S. (2011). "Coordenação nas Atividades Colaborativas em Ambientes de Aprendizagem - uma Avaliação na Implementação de Arquiteturas Pedagógicas. RENOTE. Revista Novas Tecnologias na Educação.

Vygotsky, L. S. (1978). Mind in Society: the development of higher psychological processes. Massachusetts: Harvard University Press. 